\title{
Abordando la informalidad urbana desde el taller de diseño: Mejoramiento del espacio público en barrios populares, Bogotá
}

\author{
Addressing urban informality since the design studio: \\ Improving public space in informal settlements in Bogotá
}

\section{Resumen}

Autores:

Jaime Hernández-García hernandez.j@javeriana.edu.co

Martha Inés Sierra-Moncada martha.sierra@javeriana.edu.co

Departamento de Estética. Pontificia Universidad Javeriana. Bogotá

Colombia

Recibido: 16/Mar/2019 Aceptado: 19/Nov/2019
A continuación, se presentan y analizan los resultados de un proyecto de investigación y de acción pedagógica en torno a la exploración -de dinámicas socio-espaciales en barrios populares de Bogotá, y las propuestas de mejoramiento del espacio público que se produjeron en un taller internacional de verano con estudiantes de Colombia y de México, donde también participaron miembros de la comunidad. El taller buscó propiciar la reflexión de los estudiantes respecto al espacio público popular y entender las dificultades y responsabilidades al enfrentarse a la ciudad informal. Esto sin mencionar el intercambio cultural con estudiantes de otros países, que favorece una visión más completa y compleja de la disciplina. El artículo discute la importancia de este tipo de ejercicios para la formación de los futuros arquitectos, que además contribuyen al pensamiento y desarrollo de amplias zonas de nuestras ciudades latinoamericanas.

Palabras clave: informalidad, espacio público, diseño, Latinoamérica, Bogotá.

\section{Abstract:}

The article presents and analyses the results of a research project and pedagogical action on the exploration of socio-spatial dynamics in informal settlements in Bogotá and the proposals of public space developed, in a summer international design studio with students from Colombia and México, in which members of the community also took part. The workshop sought to encourage students' reflection on popular public space and to understand the difficulties and responsibilities when facing the informal city. In addition to the cultural exchange with students of different countries that facilitates a more complete and complex vision of the discipline. The article argues the importance of these tasks for the training of future architects and the contribution to the thinking and development of large areas of our Latin American cities.

Keywords: informality, public space, design, Latin America, Bogotá. 


\section{Introducción}

A pesar que la arquitectura y urbanismo informal son evidentes en las ciudades de América Latina, solo recientemente su estudio se ha incorporado tímidamente a los currículos de la formación de arquitectos en la región de manera consistente (Miranda Gasull, 2017). En este sentido el presente artículo analiza los resultados de una experiencia pedagógica con estudiantes de arquitectura de la Pontificia Universidad Javeriana de Colombia y la Universidad Iberoamericana de México, en torno a la exploración de dinámicas socio-espaciales de barrios/colonias populares o informales ${ }^{1}$ en Bogotá, y las propuestas de mejoramiento del espacio público que se desarrollaron en un taller internacional de verano donde también participaron miembros de las comunidades.

Los barrios o colonias populares, o simplemente asentamientos informales, son mayoritarios en las ciudades de América Latina. Conolly (2013) afirma que constituyen el paradigma del hábitat latinoamericano, siendo una característica distintiva de las ciudades de la región, mientras Hernández y Becerra (2017) los explican como parte constitutiva de la modernidad. Se estima que más del $80 \%$ de la población en Latinoamérica vive en ciudades (UN-Habitat, 2015), con un $23,5 \%$ de la población, o 113 millones de personas, en 2012, viviendo en condiciones definidas por las Naciones Unidas para los Asentamientos Humanos (ONUHabitat, 2012) como asentamientos informales, cifra que ha ido en aumento. En Bogotá, por ejemplo, más del $50 \%$ de la ciudad ha crecido a partir de patrones de informalidad (Rueda García, 2000). La migración del campo a las ciudades en la búsqueda de mejores oportunidades económicas y sociales ha sido el motor de este crecimiento, donde además ha contribuido el conflicto armado interno que ha desplazado forzosamente a muchos pobladores rurales (Hernández-García, 2016).

Estos asentamientos han sido vistos tradicional e históricamente como un problema que necesita ser resuelto por los gobiernos, tanto en términos de proveer a la gente con vivienda, como erradicando estos desarrollos marginales que rodean las ciudades (Hernández-García, 2016). Al paso del tiempo, y aunque la percepción sobre los asentamientos populares no ha cambiado de forma sustancial, si se han reconocido otras perspectivas y entendimientos; quizás la principal es entenderlos como parte fundamental del paisaje socio-espacial de América Latina (Brillembourg y Klumpner, 2010; Hernández y Kellett, 2010; Lima y Pallamin, 2010). Igualmente, el reconocimiento de que esta forma de urbanización está intrínsecamente relacionada con la ciudad latinoamericana: "las prácticas informales de urbanización y vida urbana, coexisten con el desarrollo urbano regulado en un vasto territorio de condiciones físicas, legales y sociales contrastantes" (Lima y Pallamin, 2010, p. 39). Inclusive muchos han identificado su valor como construcción socio-espacial, como un modo alternativo de producción de espacio (Roy, 2009, p. 826). En este sentido, es necesario ver estos asentamientos como son, como parte de las ciudades, con problemas, oportunidades, e incluso con lecciones que pueden ofrecer de integridad, inclusión y diversidad (Fiori y Brandao, 2010; Hernández-García, 2012 y 2016)

En este contexto, se desarrolló entre los meses de Junio y Julio de 2017 un taller internacional de verano con estudiantes de arquitectura de las Universidades Javeriana (Colombia) e Iberoamericana (México) para estudiar el espacio público popular y hacer propuestas de mejoramiento participativas con las comunidades. El taller se dividió en dos partes, la primera en Bogotá en el barrio San Luis Altos del Cabo y la segunda, en Ciudad de México, en el pueblo originario² San Mateo Tlaltenango. En Bogotá, con el apoyo de la Fundación OASIS, organización no gubernamental que trabaja en el barrio, y en Ciudad de México con la ayuda del Consejo de Gobierno del pueblo originario. El presente artículo presenta y analiza la experiencia de Bogotá. En este sentido, el objetivo fundamental es documentar y analizar una práctica pedagógica, además de aportar al desarrollo físico-espacial de las comunidades y contribuir al entendimiento de la informalidad urbana.

El taller buscó propiciar la reflexión de los estudiantes respecto al espacio público popular y el planteamiento de propuestas de diseño, desde los atributos de sentido de lugar, disponibilidad de servicios, materiales e infraestructura, adecuación cultural, ambiental y económica. Pero en especial era importante el trabajo conjunto con las comunidades para evidenciar los problemas y las oportunidades, y construir las propuestas resultantes; trabajo que enriqueció el proceso formativo de los estudiantes no solamente en cuanto al componente académico se refiere, sino a la experiencia misma que los enfrenta a dificultades y responsabilidades al enfrentarse a la ciudad informal. Esto sin mencionar el intercambio cultural con estudiantes de otros países que favorece una visión más completa y compleja de la disciplina.

Después de una contextualización teórica, se presenta el ejercicio del taller internacional de verano Ciudades globales, retos y oportunidades de los barrios informales, discutiendo la metodología, presentado el caso de estudio en el barrio San Luis Altos del Cabo en Bogotá, pasando luego a describir y analizar los proyectos de los estudiantes, así como su entendimiento y contribución al tema. El artículo finaliza con unas conclusiones en torno a la importancia de este tipo de ejercicios en nuestras escuelas de arquitectura, que constituyen una doble apuesta: la formación de los estudiantes y la contribución al pensamiento y desarrollo de amplias zonas de nuestras ciudades latinoamericanas.

${ }^{1}$ Popular e informal se usan como sinónimos en este artículo, que, si bien tienen algunas diferencias, en general lo primero se usa más en Latinoamérica (particularmente en Colombia y México) mientras lo segundo aparece más en la literatura internacional. Por otra parte, barrios se usa en Colombia y colonias en México.

2 Término relativamente reciente en México, que identifica a las comunidades originarias de los territorios de origen prehispánico, con características sociales, culturales y simbólicas particulares (Álvarez Enríquez, 2011). Muchos de estos pueblos, como San Mateo Tlaltenango, tienen altos grados de informalidad urbana y marginalidad, que los hacen semejantes a las colonias populares. 


\section{Espacio público en los barrios populares}

Aunque pudiera argumentarse que los barrios populares o informales existen casi desde la aparición misma de la ciudad en América Latina, se empezaron a documentar y visibilizar desde los años 1960s, cuando el tema fue lo suficientemente visible para llamar la atención (Hernández-García, 2012), vinculado al crecimiento demográfico, la migración campo-ciudad y el modelo económico imperante (Miranda Gasull, 2017).

Las ciudades latinoamericanas tuvieron un rápido crecimiento, pasando en 1950 de tener menos del 40\% de población urbana, a 1990 con población en las ciudades que sobrepasaba el 70\% (Gilbert, 1998), y en la actualidad a sobrepasar el 80\% (UN-Habitat, 2015). La migración del campo a las ciudades fue el motor de este crecimiento, trayendo masivas cantidades de pobladores a los centros urbanos, ciudades que no eran capaces de acomodar a estos nuevos habitantes, forzándoles a que resolvieran por su cuenta el problema y encontrando una alternativa en los asentamientos populares.

Estos asentamientos eran ilegales, considerados marginales y problemáticos. Fue discutido $-\mathrm{y}$ en cierta medida todavía se discute- que la gente pobre permanece pobre y marginal, que son incapaces de lograr alguna mejora en sus vidas y en sus entornos físicos. Conceptualizaciones que fueran propuestas especialmente por Lewis (1963) como parte de su trabajo en México, explicando los barrios populares como lugares de pobreza y marginalidad. Esta interpretación fue, sin embargo, refutada con los trabajos de Mangin (1967) en Perú, y Perlman (1976) en Brasil, quienes argumentaron los mitos de la marginalidad, identificando muchos más aspectos de la vida social y urbana de los barrios populares, que solo temas de pobreza, marginalidad $y$ exclusión.

Para las Naciones Unidas, los asentamientos informales se caracterizan por carencia de los servicios básicos, viviendas deficientes, estructuras de construcción inadecuada e ilegal, hacinamiento, alta densidad, condiciones de vida malsanas, sitios peligrosos, tenencias inseguras, pobreza y exclusión social (UN-Hábitat, 2003). En Colombia, aparte de estas características, otros aspectos son su localización periférica, usualmente en suelos no aptos para la urbanización y/o en zonas de riesgo, con dificultades de acceso y movilidad (Sierra, 2006). Aunque son claras las carencias socio-espaciales de estos barrios, es importante resaltar también las contribuciones positivas sociales, económicas, arquitectónicas y urbanas que allí se encuentran, además de las condiciones y capacidades de las comunidades (Hernández-García, 2016; Gilbert, 2007; Robinson, 2006) En este sentido, el presente artículo entiende los barrios o colonias populares como entidades socio-espaciales donde la iniciativa y participación de los residentes en la conformación espacial de sus áreas públicas y privadas es central (Hernández-García, 2012), sin desconocer las precarias condiciones sociales y espaciales que se encuentran en muchos de estos asentamientos.
Los barrios populares pueden ser considerados como una forma de producción y construcción social del espacio. La producción del espacio es orientada en gran parte por la gente.

En una relación dialéctica con la producción, Low (1996), sostiene que el consumo del espacio es en realidad una transformación que se da "a través de los intercambios sociales, los recuerdos, las imágenes y el uso cotidiano que la gente hace del entorno material" (pp. 861862). El lenguaje, junto con su producción y uso, está intrínsecamente relacionado con las personas. En otras palabras, el lenguaje es el resultado de las interacciones físicas y simbólicas de los individuos con el espacio, y los significados atribuidos a ese lenguaje tienen un impacto sobre las personas.

La producción del espacio urbano y arquitectónico en los asentamientos informales son actos cotidianos donde se toman decisiones de diseño de manera permanente, decisiones que no necesariamente siguen patrones habituales, y que se anidan en un orden complejo (Hernández García, Niño Bernal, y Hernández-García, 2016). Muchas de estas decisiones pueden explicarse con las mismas herramientas de un proceso formal de diseño. Sin embargo, la diferencia está en quién o quiénes impulsan esas decisiones, y cómo las ponen en práctica.

La literatura evidencia que existe creatividad y una estética particular en los barrios populares (Carvajalino, 2004; García Canclini, 1989; Hernández-García, 2012; Kellett y Napier, 1995; Klaufus, 2000; Mehrotra, 2010; Miles, 2000). Estas expresiones creativas y estéticas no solamente reafirman la cultura de las comunidades, sino que también dejan impresiones y sensaciones tangibles en el entorno y lo van construyendo. En este sentido, tanto las expresiones como las actividades cotidianas que se desarrollan en los barrios populares contribuyen a darle forma.

En este contexto el espacio público surge en los barrios populares como una respuesta auto-gestionada del espacio urbano. Los principales espacios públicos del barrio popular son la calle y el parque o cancha. Las calles pueden convertirse en escaleras, adaptándose a la topografía escarpada que encontramos en muchos de los barrios (Velarde Herz, 2017); el parque incluye la cancha como espacio deportivo y otras áreas recreativas, en ocasiones espacios pavimentados y zonas verdes.

Las grandes áreas pavimentadas encontradas en otras partes de la ciudad - conocidas como plazas heredadas de la época colonial- no existen en los barrios. La plaza se ha transformado en el parque del barrio, con asociaciones y significados similares para los habitantes, populares y de importancia comunitaria, simbólica y social (Hernández-García, 2012). Las relaciones sociales y las expresiones culturales se construyen en las calles y los parques. Sus esquinas y canchas deportivas adquieren nuevos usos, y a la vez la trasformación espacial emerge. El espacio público en los barrios populares puede entenderse también como aquel donde la gente quiere tener más control, es decir, más apropiación, más libertad (Hou, 2010). Estas actividades de consumo de espacio, de apropiación y de búsqueda de libertad, también ayudan a construir una red de experiencias y conexiones con 
el lugar. Entre los usos que se observan en los espacios públicos populares están: caminar, reunirse, hablar, jugar, comer, beber y comprar. Estas actividades muestran las tendencias de las prácticas diarias relacionadas con los espacios públicos; no obstante, se podría decir que cada caso es diferente, lo cual confirma la diversidad de los asentamientos populares y sus dinámicas sociales y físicas, que pueden ser comprendidas a través de sistemas complejos (Hernández García, Niño Bernal, y Hernández-García, 2016).

El espacio público toma una especial dimensión en los barrios populares, debido a que la construcción socioespacial cotidiana en estos asentamientos urbanos comienza y termina en el espacio público. Comienza, en virtud de que el trazado de calles es lo que primero se hace, para luego definir los lotes y desarrollar las viviendas a lo largo del tiempo. Una vez las viviendas llegan a algún grado de consolidación, los ojos vuelven a ponerse en el espacio público, justamente para tratar también de mejorarlo, a partir de las iniciativas de la gente, pero también de las posibilidades y coyunturas identificadas en las oficinas públicas (Hernández-García, 2012)

\section{Taller internacional de ve- rano: Ciudades globales retos y oportunidades de los ba- rrios informales}

Los análisis que propician este artículo son resultado de taller internacional de verano Ciudades globales retos y oportunidades de los barrios informales, en su versión 2017. El taller ha planteado, en esta y en versiones anteriores, la aproximación a barrios populares como una experiencia de aprendizaje para grupos de estudiantes y como un reconocimiento del potencial inherente, existente en estas zonas de las ciudades. Se trabajó en el barrio popular San Luis Altos del Cabo, en Bogotá y en el pueblo originario San Mateo Tlaltenango en Ciudad de México. El presente artículo señala la experiencia de Bogotá, y se inscribe en la línea de trabajo con comunidades vulnerables de asentamientos informales de Bogotá de la carrera de Arquitectura de la Universidad Javeriana, línea que está vinculada con responsabilidad social universitaria, y en particular este trabajo, con las investigaciones del Departamento de Estética en torno a informalidad urbana, estética popular y participación comunitaria.

El barrio San Luis Altos del Cabo y los sectores aledaños son representativos de los sectores informales. Entre los rasgos que cabe destacar están: la localización periférica, la deficiente provisión de servicios públicos, las precarias condiciones de las viviendas, la inseguridad en la tenencia, y las difíciles condiciones socioeconómicas.

El taller parte del reconocimiento de que un gran porcentaje de las ciudades contemporáneas latinoamericanas son resultado de la autogestión y a veces autoconstrucción, por parte de sus propios habitantes (Hernández-García, 2016); y que por tanto es importante la aproximación y reflexión acerca de estas realidades, que en muchas ocasiones se han desconocido y subvalorado.

\subsection{Metodología}

La metodología desarrollada incluyó revisión documental, elaboración de cartografías sociales, y observación/ recorridos guiados por el sector

En la revisión documental se buscaba determinar las condiciones y características del sector de intervención, su historia y la relación con el resto de la ciudad. Se utilizaron fuentes oficiales, que se contrastaron y verificaron con la información recogida en la cartografía social y con la observación de campo, indagando también por las necesidades y expectativas de la comunidad. Las tres aproximaciones se complementan entre ellas y permitieron la construcción de una imagen más completa de la realidad.

La cartografía social propició, a través de la construcción de mapas, entender la concepción que los habitantes tienen del territorio y sondear temáticas específicas, en este caso, la existencia y uso del espacio público dentro del barrio. Se considera además una herramienta de planificación social (Habegger y Mancila, 2006), pues facilita la construcción colectiva de conocimiento, la autorreflexión y el empoderamiento de los participantes (Barragán, 2016).

Por su parte, la observación facilitó una comprensión holística del lugar, el contexto y la situación de estudio, utilizando los cinco sentidos del observador (Kawulich, 2006) y permitiendo la aproximación a las actividades que las personas desarrollan espontáneamente en el espacio (Dewalt y Dewalt, 2011). Al respecto se recomendó a los estudiantes prestar atención al espacio público, los actores presentes en él y sus interacciones, entendiendo a los habitantes como colaboradores en el proceso de construcción de la información.

Es de destacar que la combinación de instrumentos permitió a los estudiantes aproximarse de manera ágil a un sector ajeno y desconocido con una buena proporción de realidad, según reconocieron los habitantes en una visita posterior en la que se socializaron los resultados. También fue posible la verificación de información desde diferentes fuentes e instrumentos, lo que permitió la triangulación de los mismos.

La información recolectada fue estudiada y organizada para producir un diagnóstico preliminar en el que cada grupo de estudiantes identificó los retos y oportunidades del sector que le correspondió. En un primer avance se caracterizó el sector, los principales problemas y las posibilidades de intervención. Posteriormente, los grupos definieron unas estrategias generales y una acción específica de intervención. Los resultados fueron sintetizados en planimetrías de análisis que permitieron a su vez definir áreas de trabajo para cada grupo e intervenciones físico-espaciales concretas. 


\section{San Luis Altos del Cabo}

\subsection{Caracterización del barrio}

La ciudad de Bogotá se distribuye políticoadministrativamente en 20 localidades y normativamente estas localidades se subdividen en Unidades de Planificación Zonal (UPZ), que determinan la norma y el tratamiento a aplicar en un determinado sector.

El barrio San Luis Altos del Cabo (o simplemente San Luis) se sitúa en la Localidad de Chapinero y concretamente en la UPZ 89, denominada San Isidro-Patios, a la que le corresponde el tratamiento de mejoramiento integral. Ambas se localizan sobre el borde nororiental de la ciudad. La UPZ está rodeada de suelos de protección ambiental que la separan del resto del perímetro urbano (Figura 1).

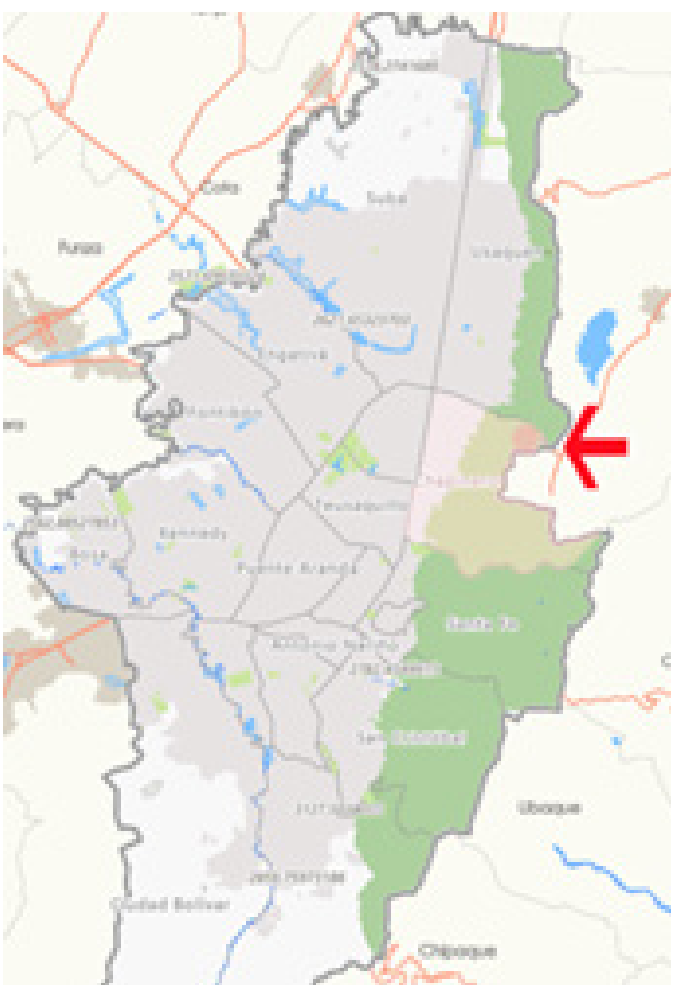

Figura 1: Localización del barrio San Luis Altos del Cabo en Bogotá.

Fuente: Elaboración de los autores sobre cartografía de mapas Bogotá https://mapas.bogota.gov.co/

En este sentido el barrio, junto con La Esperanza y San Isidro, los otros barrios de la UPZ, constituyen una isla de urbanización dentro un entorno natural. En cuanto a conexión con la ciudad, se llega al sector por la vía que lleva de Bogotá al municipio de La Calera, de la cual se deriva un ramal, unos pocos kilómetros antes del peaje, para el sector.

Topográficamente es una zona de pendiente por encima del $10 \%$, llegando en algunos casos hasta el $40 \%$, localizada entre los 2900 y los 3200 metros sobre el nivel del mar por lo que algunas áreas corresponden al

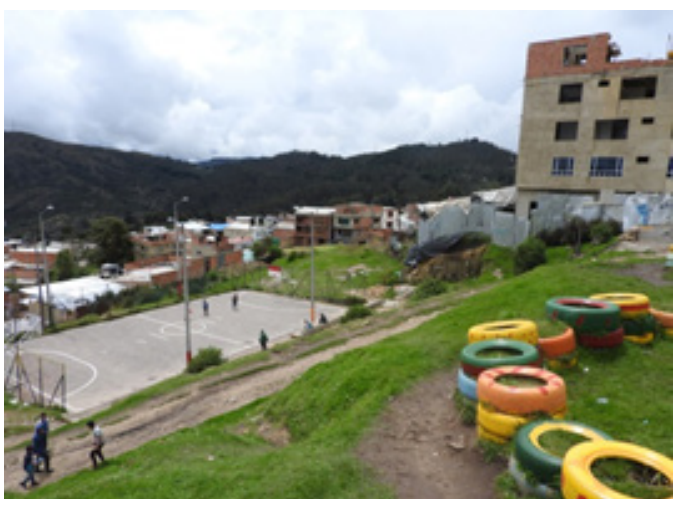

Figura 2: Barrio San Luis Altos del Cabo, Bogotá. Zona de la cancha.

Fuente: Martha Inés Sierra M.

páramo (Figura 2). Por esto último, es un área rica en cuerpos de agua, lo que en principio facilitó la ubicación de las primeras familias que llegaron al sector, hacia los años 60 del siglo XX:

[...] Las familias que llegaron, se unieron con los campesinos para mejorar las condiciones de vida y luchar por la ausencia de servicios

[...] Encontraron en la quebrada de Morací una fuente suficiente que tenía capacidad

[...]. Así que se hicieron los pozos domiciliarios con sus respectivas tuberías [...]" (Alcaldía de Chapinero, 2017, p. 2).

Esta localización determinó y determina una condición de provisión de servicios, aún hoy, precaria, especialmente en lo concerniente al agua potable, una de las grandes deficiencias del sector. Tampoco se cuenta con el servicio de gas y las rutas de transporte público, que hasta hace poco se están conectando de manera formal con el barrio. En lo que respecta a las viviendas, teniendo en cuenta que los primeros habitantes Ilegaron hace más de 60 años, muestran hoy diferentes niveles de consolidación, desde algunas precarias y realizadas en materiales transitorios, hasta viviendas de 3 y 4 pisos en bloque y cemento. Muchas de ellas conservan un aire rural, recordatorio del origen de la mayoría de sus habitantes iniciales.

En cuanto a la condición socioeconómica del barrio (Alcaldía de Chapinero, 2017), se encuentran 1471 familias conformadas por 5744 individuos. En su composición predominan los adultos entre los 27 y 59 años con cerca del 38\% de la población, seguidos de los jóvenes con el 37\%. El ingreso mensual de los núcleos familiares es menos de un salario mínimo; el subempleo es una de las constantes de la población y las ventas ambulantes la principal actividad económica. El mismo documento resalta que también "existe población que recibe entre 1 y 2 salarios mínimos, pero muchas de estas familias están conformadas por más de 5 integrantes con un único proveedor, siendo insuficiente el salario para la manutención del hogar". (Alcaldía de Chapinero, 2017, p.22).

\subsection{Los proyectos}

Para el desarrollo del taller se organizaron cuatro grupos, tanto para la aplicación de los instrumentos de 
recolección de información, como para la formulación de las estrategias generales. En cada uno de estos grupos se llevó a cabo al menos una cartografía social, un recorrido de observación, y también hicieron la correspondiente revisión documental para producir un plano diagnóstico identificando los retos y las oportunidades del sector.

Entre los grandes retos identificados están: la superación de las dificultades de movilidad dadas por la topografía pendiente y la relación que tradicionalmente han establecido los habitantes con los elementos naturales. En el caso de los cuerpos de agua, por ejemplo, son utilizados en su mayoría para el vertimiento de basuras y aguas servidas, lo que genera áreas de inseguridad y zonas que se utilizan de manera no deseable, como el consumo de sustancias psicoactivas por parte de los jóvenes.

En relación al potencial del sector se resalta la riqueza natural, presente en varios cuerpos de agua, una zona de reserva aledaña a los barrios y la vista sobre la ciudad, debido a su privilegiada posición.

En cuanto a lo humano, se evidencia el arraigo y compromiso de los habitantes con su sector, sus conocimientos agrícolas, así como varias expresiones culturales entre las que destacan el grafiti y el teatro, que se han manifestado gracias a los grupos culturales que funcionan en el barrio.

Las intervenciones (Figura 3) se orientaron a garantizar una mayor provisión de espacio público a través de la utilización de espacios residuales (grupo 1), la optimización de los espacios públicos existentes (grupo 3 y 4) y el desarrollo de nuevas formas de espacio público a través del uso del interior de las manzanas, aprovechando el estado incipiente de consolidación de las mismas (grupo 2).

Los cuatro grupos plantearon la inclusión de las zonas de ronda y reserva como parte de sus recorridos, bien generando circulaciones aledañas a los cuerpos de agua para garantizar más conexión y a su vez propiciar mayor seguridad para el uso de estos espacios, o bien convirtiéndolos en la culminación de los diferentes ejes propuestos. En esto se ve el interés por modificar la relación tradicional de los habitantes con estos espacios, y en consecuencia el uso que se hace de los mismos hasta este momento.

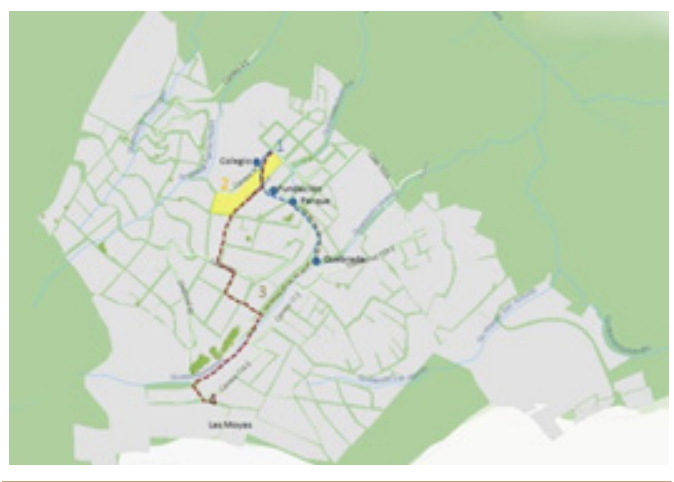

Figura 3: Mapa de las intervenciones.

Fuente: Elaboración de los autores sobre cartografía de mapas Bogotá https://mapas.bogota.gov.co/

\section{Propuestas de mejora- miento del espacio público}

El grupo $1^{4}$, remansos urbanos, plantea un recorrido de unos 400 metros cerca de los puntos clave del barrio, tales como el colegio, el parque y la fundación Un oasis en la montaña, a lo largo del cual desarrolla cuatro puntos de interés en espacios no construidos aún, considerando los aspectos natural, cultural, social y agrícola para generar zonas de permanencia para el encuentro de diferentes grupos humanos del sector. Se concentra en dos intervenciones: cultivos inclusivos y franjas programáticas.

En el proyecto de cultivos inclusivos se identifica un área con potencial agrícola, con la premisa de que las actividades de siembra, cuidado y comercialización de productos sirvan como potenciadores de la integración social de los habitantes y la disminución de la percepción de inseguridad, por la presencia de los cultivadores y la generación de zonas de permanencia.

En franjas programáticas los estudiantes proponen la intervención de un espacio cercano al colegio en el que se puedan llevar a cabo actividades como talleres de apoyo a las huertas del proyecto anterior, proyecciones de cine, presentaciones teatrales y actividades sedentes en contacto con la naturaleza apoyados por mobiliario fabricado a través de la reutilización de elementos como las estibas (Figura 4).

\section{SISTEMA DE REMANSOS}

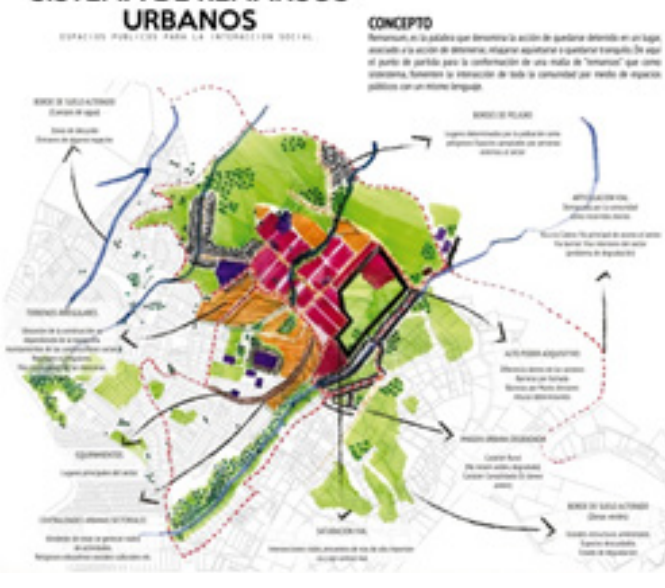

Figura 4: Foto del proyecto grupo 1 Fuente: Estudiantes del taller

El grupo $2^{5}$, Convergiendo caminos, lleva la formulación de espacio a la posibilidad de modificar la estructura urbana existente, aprovechando el bajo nivel de consolidación de algunas manzanas en las cuales hay varios lotes aún sin construir. Esto permite mayor permeabilidad peatonal y la generación de espacio público. Este grupo vincula los

${ }^{3}$ El salario mínimo para el año 2017 en Colombia correspondía 737.717 pesos colombianos, unos 245 dólares estadounidenses

${ }^{4}$ Estudiantes Ruizorduz, Granados, Rojas, Vargas, Cipagauta, Sarria, Salame, Levy, Jeitani y Avelar. 
espacios a una de las grandes necesidades del sector: la provisión de agua. Los proyectos desarrollados fueron: senderos emergentes y umbrales urbanos.

La estructura urbana, resultado en buena medida de las condiciones topográficas del lugar, propicia vías con potencial para su tratamiento peatonal por su discontinuidad, sinuosidad y pendiente, entre otras razones. Los estudiantes reconocen en estos rasgos el potencial para la generación de espacio público, ejes de movilidad peatonal exclusiva estrechamente relacionados con las viviendas, y la posibilidad de localizar artefactos atrapa-niebla, propicios por la altura y condiciones climáticas existentes, como fuente alternativa de provisión de agua.

De igual manera, se aprovecha el incipiente nivel de consolidación de las manzanas del sector, en las que se encuentran algunas pocas construcciones con varios predios vacíos. En este caso la propuesta es generar espacio público al interior de las manzanas haciendo que las viviendas existentes abran sus fachadas correspondientes hacia este espacio (Figura 5).

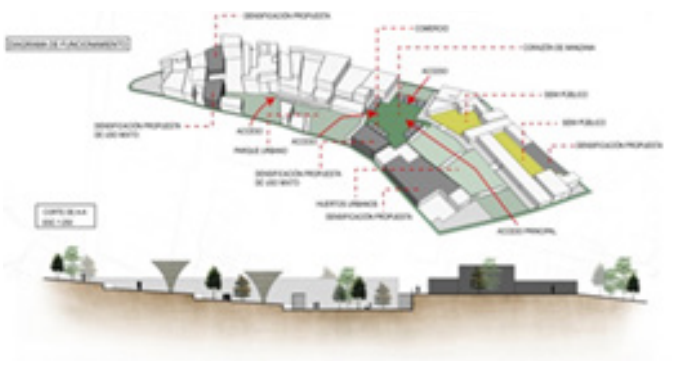

Figura 4: Foto del proyecto grupo 1

Fuente: Estudiantes del taller.

El grupo $3^{6}$, Reinserción comunitaria, centra sus reflexiones en aspectos de accesibilidad y funcionalidad para propiciar más y mejores actividades por parte de la población, incluyendo adultos mayores, en dos espacios, actualmente en condiciones precarias.

Uno de los espacios públicos principales existentes es el parque. Sin embargo, sus condiciones de accesibilidad y uso debido a la pendiente del terreno son limitantes, especialmente para adultos mayores y niños pequeños (Figura 2). El grupo propone La pedrera, intervención que facilitaría la movilidad y aprovechamiento de la pendiente en la formación de un escenario para presentaciones artísticas, cuyo diseño propicia espacios de permanencia y rompe las barreras de movilidad del lugar.

De igual manera, en este proyecto se trabaja con las condiciones del terreno para generar un recorrido con espacios de permanencia a lo largo del mismo. El recorrido se diseña enfocado a la población de

${ }^{5}$ Estudiantes Sabogal, Román, Pérez, Galván, Mancera, Vargas, Ávila De los Ríos, Mejía y Corredor

6 Estudiantes Escobar, Valbuena, Ramírez, Ramírez, Maldonado, Córdoba, Frías, Hernández, Jiménez y Castañeda.

Estudiantes Biehl, Fernández, Mustri, Prada, Martín, Figueroa, Blancas, Yahia, Obregón y Ricaurte. adultos mayores, niños menores y perros, siendo parte importante del diseño la utilización de las especies vegetales propias del lugar (Figura 6).

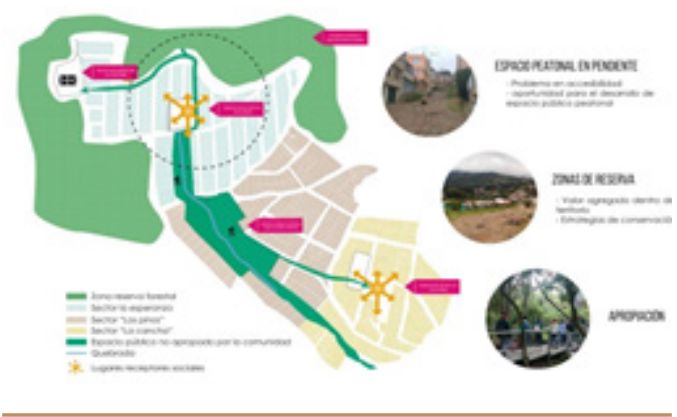

Figura 6: Foto del proyecto grupo 3

Fuente: Estudiantes del taller.

El grupo 47 , Turisteando, identificó a partir de su propia experiencia en el recorrido por el sector, el atractivo turístico que puede tener para personas ajenas al mismo, dentro del que se destacan el paisaje natural y el construido, este último por la singularidad resultado de su proceso de formación. De igual manera, expresiones culturales propias del lugar como los grafitis, murales $y$ los lugares de memoria para los habitantes del barrio se aprovechan para el desarrollo de dos circuitos turísticos (Figura 7)

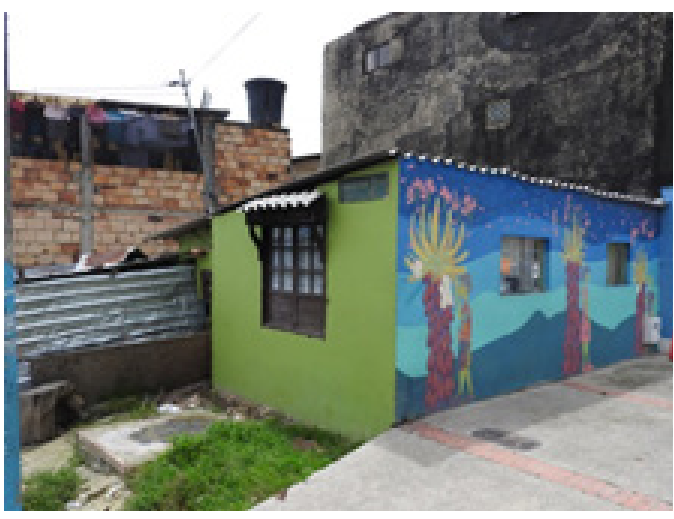

Figura 7: Arte urbano en el sector

Fuente: Martha Inés Sierra M.

En la propuesta Circuito turístico San Isidro, el grupo de estudiantes identifica puntos de interés que van desde lugares significativos para la población, como La Virgen, La Fundación, El Mirador, entre otros, que les permiten delinear un recorrido por sitios de interés con potencial para atraer a personas ajenas al sector. Esto se fortalece con la intervención en andenes y fachadas, y una propuesta de señalética.

Por su parte, el corredor verde Morací, aprovecha la zona de reserva forestal que rodea el sector y un sendero ecológico existente, no obstante, poco utilizado a pesar de encontrarse en buenas condiciones para la movilidad y uso por parte de los habitantes. Se apoya en el Programa Aulas Ambientales de la Secretaría de Ambiente buscando la apropiación social del territorio a través de acciones pedagógicas (Figura 8). 


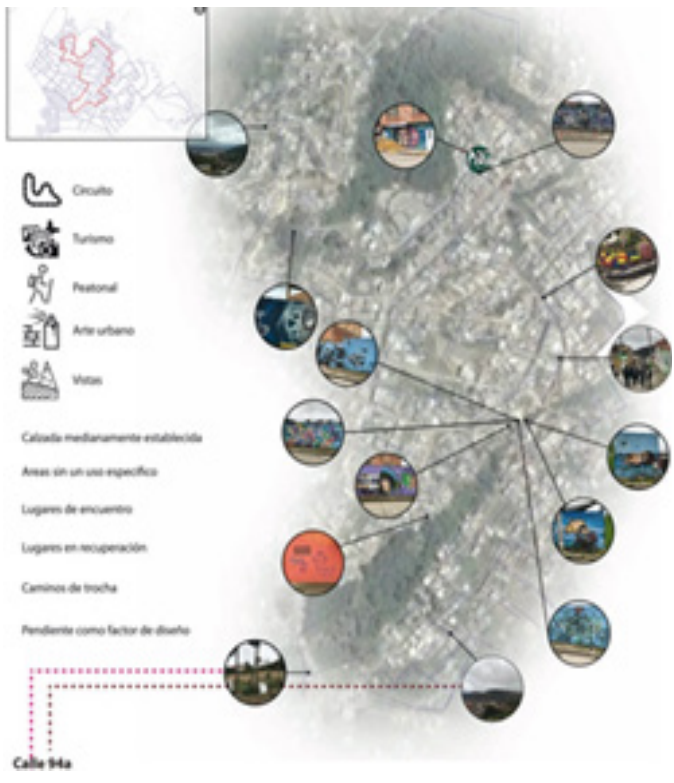

Figura 8: Foto del proyecto grupo 4

Fuente: Estudiantes del taller

Como se puede ver en las propuestas, los grupos se concentraron en los componentes de accesibilidad e intersubjetividad (Kohn, 2004), propiciando más apropiación (Hou, 2010). Es decir, las intervenciones se enfocaron en modificar la relación de los habitantes con espacios, en la mayoría de los casos existentes, especialmente en lo concerniente a garantizar y facilitar el uso, en función de las particularidades identificadas en la población.

Las propuestas tienen en común soluciones diferentes a las que se aplicarían en otros sectores de la ciudad valorando las particularidades del barrio popular y retomando el pensar-actuar alternativo de los grupos humanos presentes en estos sectores.

Desde esta perspectiva no solo constituyen respuestas acordes con las condiciones y características del barrio, sino que además se les reconoce un potencial para pensar la producción de espacio público desde perspectivas alternativas que pueden usarse para repensar la ciudad formal.

\section{Conclusiones}

La puesta en marcha del taller internacional de verano Ciudades globales retos y oportunidades de los barrios informales pone de manifiesto varios aspectos acerca de los procesos formativos en las escuelas de arquitectura, no solo en cuanto al tema de trabajo, sino también de la metodología y el marco conceptual.

Tanto para los estudiantes colombianos como para los mexicanos, aproximarse a una realidad distinta a la que usualmente desarrollan en sus ejercicios de aprendizaje de la arquitectura, constituía un reto. Y para los profesores, aunque expuestos al tema desde hace muchos años desde su quehacer principalmente investigativo, era también retador confrontar la experiencia desde la docencia y con las comunidades.

Quizás uno de los aspectos más importantes fue la aproximación a sectores de la ciudad que no suelen ser conocidos, visitados ni reconocidos cuando se habla de la ciudad latinoamericana, particularmente para los arquitectos. Se sabe, desde luego, de la existencia de los sectores informales, e incluso se puede intuir que constituyen una buena porción de la ciudad, pero no se les suele reconocer, y en general la visión predominante es que son áreas pobres, con muchos problemas, que deberían desaparecer para dar paso a una ciudad moderna. Esta visión es rápidamente controvertida cuando se empieza a explorar el tema y se confirma una vez se hacen las primeras visitas al barrio informal; existe pobreza, también ideas y realizaciones, así como comunidades comprometidas con el desarrollo socioespacial de sus asentamientos.

Desde lo metodológico, la aplicación de los instrumentos, el contacto con los habitantes del sector, así como el conocimiento de primera mano del mismo, permitieron que los estudiantes tuvieran una mirada amplia acerca de los barrios populares. Pasaron de observar solo los problemas y deficiencias a identificar la riqueza, espacial y humana, presente en la zona de trabajo, y especialmente las diferencias con el resto de la ciudad y el potencial inherente a ellas. Se dieron cuenta también de la gran necesidad de la arquitectura en estos lugares, y el importante papel del arquitecto en estas comunidades.

De manera particular, en el trabajo en el barrio San Luis se destacó el potencial turístico asociado a la riqueza natural del sector, al aprovechamiento de la topografía como reto, pero también oportunidad para el diseño de espacio público, la tradición agrícola y otros conocimientos de los habitantes para su aprovechamiento en el tipo de proyectos propuestos, como el caso de las huertas. Los estudiantes entendieron que, de la mano de la gente, pueden entender mejor el espacio, y también con ellos pueden generar mejores y más exitosas propuestas.

Este tipo de intervenciones no solo aplicarían para el barrio en cuestión sino para otros de rasgos similares, pues suele ser común la falta de espacio público cualificado. Así como los retos topográficos propios de sectores periféricos y ubicados en alta montaña, asi como la relación no conflictiva con elementos naturales, como cuerpos de agua o zonas de reserva ambiental.

De igual manera, las estrategias aplicadas en función de una mayor provisión de espacio público a través de la utilización de espacios residuales, la optimización de los espacios públicos existentes y el desarrollo de nuevas formas de espacio público, exclusivas para estos asentamientos, pueden ser replicables en otros sectores.

Más allá de cuestiones puntuales, se reconoce que trabajar en la informalidad implica una aproximación más flexible, capaz de adecuarse a los descubrimientos, capaz de improvisar si es necesario cuando las cosas no resultan exactamente como se esperaban, y es que la velocidad de la informalidad es diferente a la de la ciudad formal y consolidada, tanto en el uso cotidiano como en su construcción a lo largo del tiempo y su 
capacidad de transformación, aspectos que exigen capacidad de adaptación. En este sentido, se destaca la validez y bondades que los estudiantes reconocen en e diagnóstico participativo a través de los instrumentos utilizados. Estos les permitieron reconocer la importancia del espacio público para los habitantes de los sectores populares y también algunas de las formas creativas y diferentes de utilización que tienen del mismo.

Pedagógicamente, exponer a los estudiantes a este tipo de escenario y realidades los obliga a salirse del molde y de las soluciones previstas, los obliga a pensar diferente, más allá de los esquemas de habitación y de ocupación de espacio que ellos hayan vivido en su propia experiencia arquitectónica y urbana. Eso enriquece profundamente a través del desarrollo de habilidades, así como una sensibilización hacia el otro y sus necesidades. Se resalta que los proyectos no solo enfatizaron en el diseño de espacio, sino también en la relación y/o transformación que un diseño puede producir en la relación-percepción del habitante con el espacio.

El taller logró tocar a los estudiantes, sensibilizarlos con las ciudades donde vivimos; logró poner de manifiesto que hay otros temas que requieren la atención del arquitecto, y quizás son más apremiantes en el contexto de la ciudad latinoamericana. Pensamos que ejercicios como estos son importantes en nuestras escuelas de arquitectura, pues, aunque existen, son de cierta forma marginales, como el tema mismo, ya que seguimos formando a un arquitecto que diseña para la minoritaria parte formal de la ciudad, o para Europa o Estados Unidos.

Por último, aproximaciones de esta naturaleza ilustran campos de investigación aún por profundizar alrededor de las prácticas habitacionales de los pobladores, tales como los patrones de ocupación de los predios y la progresividad del espacio público.

Cómo citar este artículo/How to cite this article: Hernández-García, J. y Sierra-Moncada, M. I. (2020). Abordando la informalidad urbana desde el taller de diseño: Mejoramiento del espacio público en barrios populares, Bogotá. Estoa. Revista de la Facultad de Arquitectura y Urbanismo de la Universidad de Cuenca, 9(17), 69-78. doi: 10.18537/est.v009.n017.a06

\section{Referencias bibliográficas}

Alcaldía de Chapinero. (2017). Lectura de realidades, territorio social San Isidro Patios. Bogotá, Colombia. Recuperado de http://old.integracionsocial.gov.co/ anexos/documentos/1_entidad/gsi/2_chapinero_ lectura_de_realidades_san_isidro.pdf

Álvarez Enríquez, L. (2011). Pueblos Urbanos. Identidad Ciudadanía y Territorio en la Ciudad de México. Ciudad de México, Mexico: Universidad Nacional de MéxicoCentro de Investigaciones Interdisciplinarias en Ciencias y Humanidades.

Brillembourg, A. y Klumpner, H. (2010). Roles of Engagement: Caracas and the Informal City. En F. Hernández, P. Kellett y L. Allen (Eds.), Rethinking the Informal City: Critical Perspectives from Latin America. Nueva York, Estados Unidos: Berghahn Books.

Barragán, D. (2016). Cartografía social pedagógica: entre teoría y metodología. Revista Colombiana de Educación (70), 247-285

Carvajalino, H. (2004). Estética de lo Popular: Los Engalles de la Casa. Serie Ciudad y Hábitat, (11), 103-123.

Conolly, P. (2013). La ciudad y el hábitat popular: Paradigma latinoamericano. En B. Ramírez y E. Pradilla (Eds.), Teorías sobre la ciudad en América Latina, Volumen II. Ciudad de México, México: Universidad Autónoma Metropolitana.

Dewalt, K. y Dewalt, B. (2011). Participant observation: A guide for fieldworkers. Lanham, Estados Unidos: Rownam y Littlefield.

Fiori, J. y Brandao, Z. (2010). Spatial Strategies and Urban Policy: Urbanism and Poverty Reduction in the Favelas of Rio de Janeiro. En F. Hernández, P. Kellett y L. Allen (Eds.), Rethinking the Informal City: Critical Perspectives from Latin America. Nueva York, Estados Unidos: Berghahn Books.

García Canclini, N. (1989). Culturas Híbridas. México D.F., México: Editorial Grijalbo.

Gilbert, A. (1998). The Latin American City. Londres, Inglaterra: Latin America Bureau.

Gilbert, A. (2007). The Return of the Slum: Does Language Matter? International Journal of Urban and Regional Research (31), 697- 713.

Habegger, S. y Mancila, I. (2006). El poder de la Cartografía Social en las prácticas contrahegemónicas o La Cartografía Social como estrategia para diagnosticar nuestro territorio. Revista Araciega, 14. Recuperado de handle/123456789/365 http://beu.extension.unicen.edu.ar/xmlui/

Hernández, F., y Becerra, A. (2017). Marginal Urbanisms: Informal and Formal Development. En Cities of Latin America. Cambridge, Reino Unido: Cambridge Scholars Publishing 
Hernández, F. y Kellett, P. (2010). Re-Imagining the Informal Settlements in Latin America. En F. Hernández, P. Kellett y L. Allen (Eds.), Rethinking the Informal City: Critical Perspectives from Latin America. Nueva York, Estados Unidos: Berghahn Books.

Hernández García, I., Niño Bernal, R. y HernándezGarcía, J. (2016). Creación e Innovación como proceso evolutivo abierto en los mundos virtuales inmersivos. En I. Hernández (Ed.), Estética de los mundos posibles, inmersión en la vida artificial, las artes y las prácticas urbanas. Bogotá, Colombia: Editorial Pontificia Universidad Javeriana.

Hernández-García, J. (2012). Espacios Públicos en Barrios Informales: Producción y Uso, entre lo Público y lo Privado. México DF., México: INFONAVIT y UAEMex.

Hernández-García, J. (2016). Hábitat popular, ¿̇un modo alternativo de producción de espacio para América Latina? En I. Hernández (Ed.), Estética de los mundos posibles, inmersión en la vida artificial, las artes y las prácticas urbanas. Bogotá, Colombia: Editorial Pontificia Universidad Javeriana.

Hernández-García, J. (2017). Underlying Language and Meaning of Informality. En F. Hernández y A. Becerra (Eds.) Marginal Urbanisms: Informal and Forma Development in Cities of Latin America. Cambridge, Reino Unido: Cambridge Scholars Publishing.

Hou, J. (2010). Insurgent public space. Guerrilla Urbanism and the remaking of contemporary cities. Londres, Reino Unido: Routledge.

Kawulich, B. (2006). La observación participante como método de recolección de datos. Qualitative Socia Research Journal, 6(2).

Kellett, P. y Napier, M. (1995). Squatter Architecture? A Critical Examination of Vernacular Theory and Spontaneous Settlement with Reference to South America and South Africa. Traditional Dwellings and Settlements Review, VI(II), 7-24.

Klaufus, C. (2000). Dwelling as Representation: Values of Architecture in an Ecuadorian Squatter Settlement. Journal of Housing and the Built Environment, (15), 341365.

Kohn, M. (2004). Brave New Neighborhoods: The Privatization of Public Space. Nueva York, Estados Unidos: Routledge.

Lewis, O. (1963). The Children of Sanchez: Autobiography of a Mexican Family. Nueva York, Estados Unidos: Vintage Books.

Lima, Z. y Pallamin, V. (2010). Informal Practices in the Formal City: Housing, Disagreement and Recognition in Downtown Sao Paulo. En F. Hernández, P. Kellett y L. Allen (Eds.), Rethinking the Informal City: Critical Perspectives from Latin America. Nueva York, Estados Unidos: Berghahn Books.
Low, S. (1996). Spatializing Culture: The Social Production and Social Construction of Public Space in Costa Rica. American Ethnologist, (23), $861-879$

Mangin, W. (1967). Latin American Squatter Settlements: A Problem and a Solution. Latin American Research Review 2(3), 65- 98.

Mehrotra, R. (2010). Foreword. En F. Hernández, P. Kellett y L. Allen (Eds.), Rethinking the Informal City: Critical Perspectives from Latin America. Nueva York, Estados Unidos: Berghahn Books.

Miles, M. (2000). The Uses of Decoration. Essays in the Architecture Every day. Chichester, Nueva York, Estados Unidos: Wiley.

Miranda Gasull, V. (2017). El hábitat popular. Algunos aportes teóricos de la realidad habitacional de sectores desposeídos. Revista Territorios, (36), 217-238.

ONU-Habitat. (2012). Estado de las Ciudades en América Latina y el Caribe. Rumbo a una Nueva Transición Urbana. Nairobi: Programa de las Naciones Unidas para los Asentamientos Humanos. Nairobi, Kenia: ONU-Habitat.

Perlman, J. (1976). The Myth of Marginality: Urban Poverty and Politics in Rio de Janeiro. Berkeley, Estados Unidos: University of California Press.

Rueda García, N. (2000). La ciudad que no conocemos. Cien años de arquitectura en Colombia. XVII Bienal de Arquitectura. Bogotá, Colombia: Sociedad Colombiana de Arquitectos.

Robinson, J. (2006). Ordinary Cities, Between Modernity and Development. Londres, Reino Unido: Routledge.

Roy, A. (2009). Urban Informality: Toward an Epistemology of Planning. Journal of the American Planning Association, (71), 147-158.

Sierra, M. (2006). El programa de mejoramiento de vivienda. Bogotá, Colombia: Universidad Nacional de Colombia.

UN-Habitat. (2003). The Challenge of Slums. Globa Report on Human Settlements 2003. London, Reino Unido: UN-Habitat.

UN-Habitat. (2015). International Guidelines on Urban and Territorial Planning. Nairobi, Kenia: UN-Habitat.

Velarde Herz, F. (2017). El espacio público en la ciudad popular: la vida entre laderas. Bulletin de L'Institut Francaise D'Etudes Andines. 46, (3), 471-488. 